\title{
Achieving Maximum System Capacity in Multiple- High Altitude Platforms through Interference Alignment
}

\author{
Sudheesh P. G.*, Maurizio Magarini ${ }^{\dagger}$, P. Muthuchidambaranathan* \\ ${ }^{*}$ Department of Electronics and Communication Engineering \\ National Institute of Technology, 620015 Tiruchirappalli, India \\ Email:pgsudheesh@gmail.com,muthuc@nitt.edu \\ ${ }^{\dagger}$ Dipartimento di Elettronica, Informazione e Bioingegneria \\ Politecnico di Milano, 20133 Milano, Italy \\ Email: maurizio.magarini@polimi.it
}

\begin{abstract}
High-altitude platforms (HAPs) provide higher throughput to the users in ground unlike conventional terrestrial communication. Various capacity improvement techniques with multiple HAPs has been suggested such as cellular architecture and virtual multiple-input multiple-output (V-MIMO) systems. Maximizing system capacity in overlapped coverage area is still an open research area. In this scenario, we propose a system that maximizes capacity between two HAP and two ground stations. The main advantage of our proposed scheme is that each HAP can send data to the receiver without any cooperation. Restriction of precise beamforming of HAP antennas for cellular like architecture is removed with our scheme. The bit error rate performance of the proposed scheme in Rural and Urban scenarios are compared. In addition, the system capacity at various signal-to-noise ratio (SNR) values and Rician factors for multiple HAP configurations are analyzed. The simulation results validate the improvement in capacity compared to the existing schemes.
\end{abstract}

\section{INTRODUCTION}

It is evident that the demand in wireless communication for higher data rate and higher transmission reliability is increasing day by day. Satellite and terrestrial communications are two well known schemes in the present days. Though satellites provides wider coverage area and service to even remote user, they are not capable of delivering high spectral efficiencies as provided by the conventional terrestrial infrastructure [1]. Terrestrial communication fails to provide reliable service throughout the service area. A possible solution is to use highaltitude platforms (HAPs), which are either airships or planes that operates in the stratosphere, $17-22 \mathrm{~km}$ above the ground. This unique position offers a significant link budget advantage compared with satellites and a much wider area of coverage than terrestrial [1].

Recent works have considered integration of HAP as a mobile station in LTE and 5G networks [2]. Such a network architecture would decrease the cost of deployment considerably [3]. In [4], the effect of HAP based transmission in cellular systems is considered, where a single HAP serves multiple ground stations located at different cells. Associating HAP with centralized networks also result in providing highdata rate to the users [5]. Recently researchers considered networking of multiple HAPs to provide even higher data rate by combining several HAPs. Such an interconnection between several HAPs are carried out by Interplatform Link (IPL). Networked configurations of several HAPs via IPLs can be considered as a virtual MIMO (V-MIMO) [6]-[8] and by doing so it is possible to exploit the advantages of distributed antenna techniques. It is worth noting that the antenna spacing in VMIMO is so high and this provides good correlation properties.

However, having IPL between HAPs are an extra burden and a V-MIMO restricts each HAPs to transmit same information. This is not the case in real scenario, where each HAP would be having separate terrestrial data or any other information. In another view, speaking of capacity, the overall capacity of the system can be maximized if each of the transmitter transmits separate information. Such an architecture where each transmitter has data to each receiver can be called as a wireless $\mathrm{X}$-network.

Unlike in [6]-[8] where authors has considered V-MIMO, in [9], [10] multiple HAPs serve a common coverage area without an IPL between each other. Such an HAP configuration would lead to an interference based system. As the user receives interference from multiple HAPs, where only a single HAP transfers data to the user, capacity of this system in limited by carrier-to-interference-plus-noise ratio (CINR). Hence from Shannon's law, $\mathrm{C}=\log (1+\mathrm{CINR})$ would bring out the effect of CINR in system capacity.

In a generalized form, at larger signal-to-noise ratio (SNR), the capacity of a network versus SNR can be approximated as

$$
C(\mathrm{SNR})=d \cdot \log (\mathrm{SNR})+\mathcal{O}(\log (\mathrm{SNR})),
$$

where $d$ defines the number of degrees of freedom (DoF) and SNR is the signal-to-noise ratio of the network. Recently DoF of X-networks [12] have been characterized. Interference Alignment (IA) have paved the way for the achievability of the Degrees of Freedom (DoF) of the MIMO X-network. The theoretical bound on the DoF region is defined as

$$
\frac{M N A}{M+N-1 / A} \leq D o F \leq \frac{M N A}{M+N-1},
$$


where $M$ and $N$ corresponds to number of transmitters and receivers, respectively, and $A$ is the number of antennas in each receiver and transmitter. In this paper we consider a $2 \times 2 \mathrm{X}$ network, which makes $M=2$ and $N=2$.

A crucial assumption to achieve IA [14]-[16] is that channel state information (CSI) is globally available at each transmitting node. Unlike terrestrial channel modelling, Airto-Ground (A-to-G) should consider four propagation mechanisms, which include free-space propagation, reflection, diffraction and scattering. Various parameters [17] such as rain attenuation, gaseous absorption and scintillation affects the free-space loss. Hence A-to-G channel can be modelled with line-of-sight (LOS) and non-line-of-sight (NLOS) connections at millimeter frequency band.

In this paper, we intend to maximize the capacity of a configuration of two HAPs with overlapping coverage area which serve two users. Hence IA is applied for the first time in HAP to Ground communication forming a $2 \times 2 \mathrm{X}$ channel for the purpose of capacity maximization. In the present scenario, where A-to-G communication is increasingly used for reliable and high data rate communication, our technique provides a better way to achieve multiple HAPs to ground station communication. Comparing with V-MIMO configuration, our technique aims at transmitting separate data from each HAP by avoiding IPLs between individual HAPS. Apart from removing the extra hardware for IPL, we also intend to remove the huge number of antennas required in the receiver. It is also possible to use directional antennas at HAPs to maximize capacity [1], but the cost incurred for developing this cell like configuration is high. On the contrary, our proposed technique does not require precise beamforming.

The paper is organized as follows. A review of system model and channel modelling is provided in Section II. In Section III a detailed explanation of the IA scheme for $2 \times 2$ user X network A-to-G channel is given. Section IV is devoted to numerical results while conclusions are drawn in Section V.

\section{System Model And Channel Modelling}

\section{A. System Model}

We consider two receiver ground stations which are served by two HAPs with overlapping coverage areas. The reference network architecture, which is investigated in this paper, is depicted in Fig. 1. Each receiver gets separate signals from each HAP forming an X-network. Each transmitter and each receiver is equipped with 3 antennas. The transmitters are assumed to be transmitting equal amount of power and the common coverage area of two HAPs corresponds to that of a metropolitan city, i.e., less than a few $K \mathrm{~m}^{2}$. It is also assumed that the transmitters and receivers transmit at a frequency band where no other HAPs or terrestrial communication exists.

\section{B. Channel Modelling}

Under terrestrial communication, the channel is modelled as Rayleigh in urban areas and Rician in suburban scenario. This is not the same for A-to-G channel [17]. Under urban conditions, A-to-G channel experiences Rician fading due to the presence of LOS path. In suburban areas a Rayleigh fading is experienced due to the presence of stronger reflected signals which are stronger than LOS.

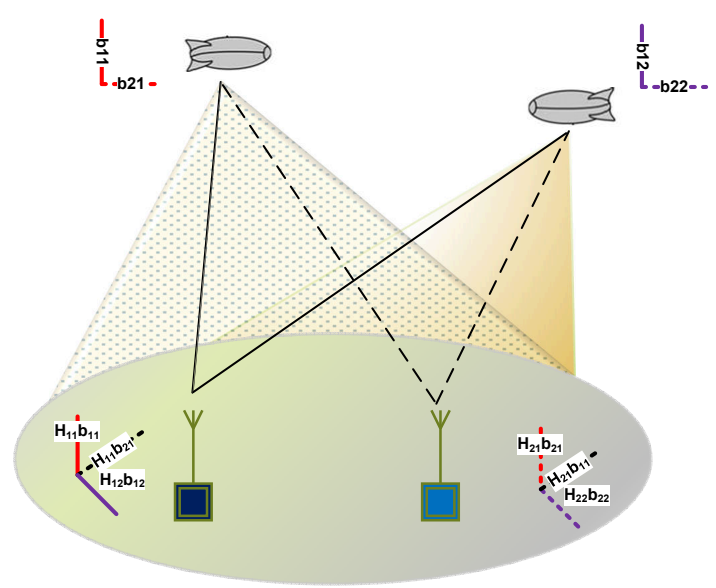

Fig. 1. Multiple HAP with overlapping coverage area

A generalized approach is to follow Rician distribution where both LOS and NLOS paths are considered. Therefore, the channel model can be represented as

$$
\mathbf{H}=\sqrt{\frac{\kappa}{1+\kappa}} \mathbf{H}_{L O S}+\sqrt{\frac{1}{1+\kappa}} \mathbf{H}_{N L O S},
$$

where $\mathbf{H}_{L O S}$ represents shadowed free space propagation loss and $\mathbf{H}_{N L O S}$ represents only the NLOS path. Hence $\mathbf{H}$ is the $N_{R} \times N_{T}$ matrix having complex fading coefficients, where $N_{R}$ and $N_{T}$ are the number of receiving and transmitting antennas. With $\sigma_{L O S}^{2}$ and $\sigma_{N L O S}^{2}$ being the power of LOS path and NLOS path respectively, the Rician factor $\kappa$ is given by [18]

$$
\kappa=\frac{\sigma_{L O S}^{2}}{\sigma_{N L O S}^{2}}
$$

In our work we consider multiple antennas at both transmitter and receiver. The MIMO channel is considered to be static and hence the LOS MIMO channel is defined as in [18]

$$
\mathbf{H}_{L O S}=\left[\begin{array}{c}
1 \\
e^{j 2 \pi \frac{d_{R}}{\lambda}} \sin \left(A o A_{R}\right) \\
\vdots \\
e^{j 2 \pi \frac{d_{R}}{\lambda}(M-1)} \sin \left(A o A_{R}\right)
\end{array}\right] \cdot\left[\begin{array}{c}
1 \\
e^{j 2 \pi \frac{d_{T}}{\lambda}} \sin \left(A o D_{T}\right) \\
\vdots \\
e^{j 2 \pi \frac{d_{T}}{\lambda}(N-1)} \sin \left(A o D_{T}\right)
\end{array}\right]^{T}
$$

where $d_{R}$ and $d_{T}$ represents the antenna spacing in the receiver and transmitter, respectively, and $A o A_{R}$ and $A o D_{T}$ represent Angle-of-Arrival at the receiver and Angle-of-Departure at the transmitter, respectively. Since the $\mathbf{H}_{N L O S}$ represents only the NLOS path, resultant NLOS MIMO channel follows Rayleigh distribution.

The presence of LOS MIMO component turns the Rayleigh distributed MIMO channel matrix to a Rician distribution as given below

$$
f_{\text {Rician }}(x)=2(1+\kappa) x e^{-(1+\kappa) x^{2}-\kappa} I_{0}(2 \sqrt{\kappa(1+\kappa) x}),
$$

where $I_{0}$ is the zeroth order modified Bessel function of the first kind and $\kappa$ is the Rician factor. 


\section{ROLE OF INTERFERENCE ALIGNMENT FOR HAP BASED TRANSMISSION}

The case where a system of two HAPs serve a common area could be useful, for example, in areas where the request of data traffic is so high that it cannot be satisfied by a single HAP. It is worth observing that to achieve higher spectral efficiency with such a system the interference between the two HAPs needs to be managed. The main novelty of our paper consists in the application of the IA principle to the case of two interfering HAPs that are equipped with multiple antennas. We consider the application of IA to a system where each transmitter or receiver node is equipped with 3 antennas. The precoding and zero-forcing vectors for IA are chosen from the knowledge of MIMO channel matrix. Hence our system can be modelled as a $2 \times 2$ MIMO $X$ channel, where each MIMO channel follows a Rician distribution due to the presence of LOS component. The choice of precoding and zero-forcing vectors is described in the following.

\section{A. Communication with two ground stations}

Each transmitting and receiving node is equipped with three antennas. The received signal vectors for user 1 and user 2 are

$$
\mathbf{y}_{1}=\mathbf{H}_{11} \mathbf{x}_{1}+\mathbf{H}_{12} \mathbf{x}_{2}+\mathbf{n}_{1}
$$

and

$$
\mathbf{y}_{2}=\mathbf{H}_{21} \mathbf{x}_{1}+\mathbf{H}_{22} \mathbf{x}_{2}+\mathbf{n}_{2}
$$

respectively, where $\mathbf{x}_{i}$ is the $3 \times 1$ signal vector transmitted by the $i$-th user, $\mathbf{H}_{j i}$ is a $3 \times 3$ channel matrix between transmitter $i$ and receiver $j$, with $i, j \in\{1,2\}$, whose elements are assumed to be independent and identically distributed (i.i.d) complex Gaussian random variables with zero mean and unit variance, and $\mathbf{n}_{j}$ is a $3 \times 1$ vector of i.i.d. complex Gaussian random variables with zero mean and variance $\sigma_{n}^{2}$. Each transmitter is intended to transmit independent messages to each of the receivers separately. The two transmitted vectors are given by

$$
\begin{aligned}
& \mathbf{x}_{1}=\mathbf{b}_{11} x_{11}+\mathbf{b}_{21} x_{21}, \\
& \mathbf{x}_{2}=\mathbf{b}_{12} x_{12}+\mathbf{b}_{22} x_{22},
\end{aligned}
$$

where $x_{j i}$ is the message to be transmitted from transmitter $i$ to receiver $j$ and $\mathbf{b}_{j i}$ is the beamforming vector associated with $x_{j i}$. By replacing (8) and (9) in (6) and (7), respectively, we have

$$
\begin{aligned}
\mathbf{y}_{1}= & \mathbf{H}_{11} \mathbf{b}_{11} x_{11}+\mathbf{H}_{11} \mathbf{b}_{21} x_{21}+ \\
& +\mathbf{H}_{12} \mathbf{b}_{12} x_{12}+\mathbf{H}_{12} \mathbf{b}_{22} x_{22}+\mathbf{n}_{1}
\end{aligned}
$$

and

$$
\begin{aligned}
\mathbf{y}_{2}= & \mathbf{H}_{21} \mathbf{b}_{11} x_{11}+\mathbf{H}_{21} \mathbf{b}_{21} x_{21}+ \\
& +\mathbf{H}_{22} \mathbf{b}_{12} x_{12}+\mathbf{H}_{22} \mathbf{b}_{22} x_{22}+\mathbf{n}_{2} .
\end{aligned}
$$

In order to satisfy the IA condition the interfering signals must span the same subspace [15]

$$
\begin{aligned}
& S P A N\left\{\mathbf{H}_{12} \mathbf{b}_{22}\right\}=S P A N\left\{\mathbf{H}_{11} \mathbf{b}_{21}\right\}, \\
& S P A N\left\{\mathbf{H}_{22} \mathbf{b}_{12}\right\}=S P A N\left\{\mathbf{H}_{21} \mathbf{b}_{11}\right\} .
\end{aligned}
$$

A possible choice of beamforming vectors is

$$
\mathbf{b}_{22}=\mathbf{H}_{12}^{-1} \mathbf{H}_{11} \mathbf{b}_{21}
$$

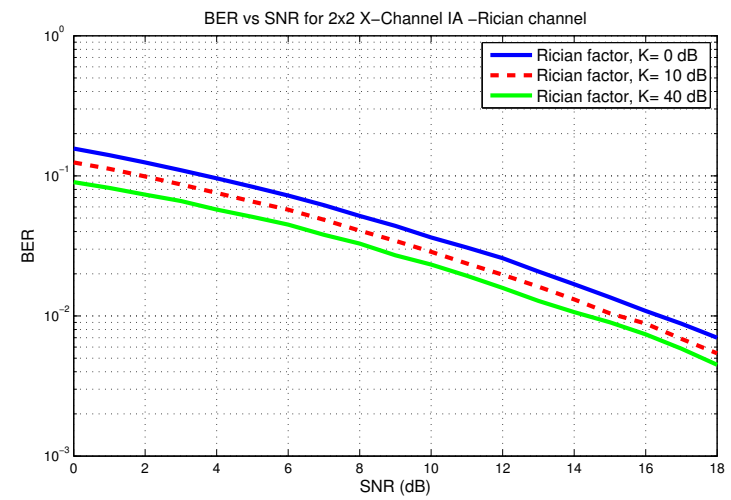

Fig. 2. BER versus SNR in case of QPSK transmission with Interference Alignment in Rician channel.

and

$$
\mathbf{b}_{12}=\mathbf{H}_{22}^{-1} \mathbf{H}_{21} \mathbf{b}_{11} .
$$

Equations (14) and (15) define the original IA solution, here termed "conventional IA", proposed in [15].

\section{NumericAl RESUltS}

The analysis is done in two phases. In the first phase, the error performance is evaluated for the IA based system as well as for the non interference system under Rician channel. The second phase describes the capacity improvement with IA under Rician channel.

The symbols $x_{11}, x_{21}, x_{12}$ and $x_{22}$ are chosen from a quadrature phase-shift keying (QPSK) constellation. Assuming an average transmitted power from each antenna equal to $P_{\text {ave }}$, the signal-to-noise ratio is defined as $\mathrm{SNR}=P_{\text {ave }} / \sigma_{n}^{2}$. As in [16], the coefficients of MIMO channels are assumed to remain constant for the entire duration of payload data transmission.

In the first phase, Monte Carlo simulations are presented to evaluate the bit error rate (BER) of the proposed IA scheme where zero-forcing detection is implemented at the receiver.

Figure 2 reports the BER performance of the IA based system for different Rician factors. The reason for performance variation is due to the effect of Rician factor in modelling the channel as in eq. (3). Hence, higher values of $\kappa$ result in predominant LOS component in received signal, forming the NLOS component less important. The BER performance is evaluated for $\kappa=0,10$, and $40 \mathrm{~dB}$. When $\kappa=0$ we have that power is equally distributed in LOS and NLOS components. For higher values of $\kappa$ increases the share of LOS component in the received signal. Hence, a Rician channel with higher $\kappa$ value offers a considerable improvement in BER performance, as reported in Fig 2. It is also important to note that the correlation between channels would be high if we take the AoA and AoD to be the same. By selecting different AoA and AoD it is possible to have good correlation properties and hence better error performance.

Figure 3 shows results of a similar experiment just like Fig. 2, except that the two HAPs are considered to be serving non overlapping areas. Hence unlike $2 \times 2 \mathrm{X}$ channel, there is no interference in this system and forms two non-interfering 


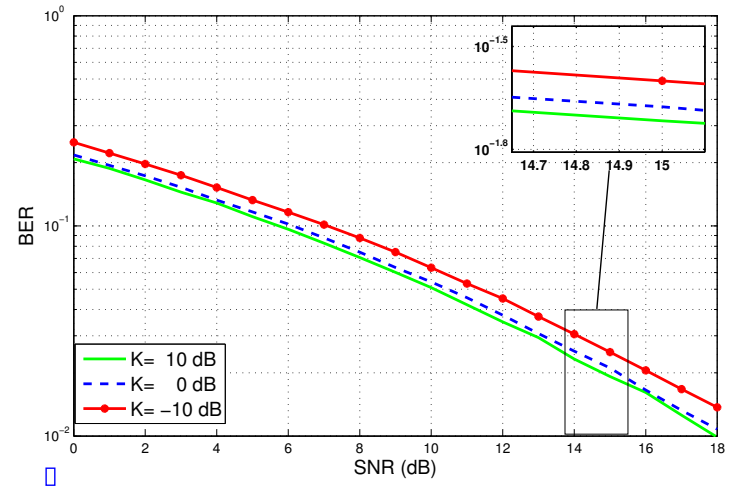

Fig. 3. BER vs SNR in case of QPSK transmission without Interference in Rician channel

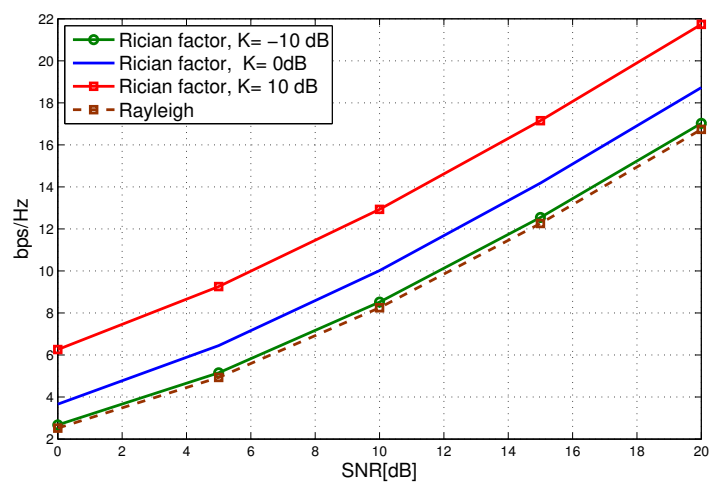

Fig. 4. Sum Rate vs SNR for MIMO with various Rician factors.

MIMO links. Unlike in Fig. 2, the curves $\kappa=10 \mathrm{~dB}$ and $\kappa$ $=0 \mathrm{~dB}$ show lesser variation, the reason for such a behavior is high correlation between the two non-interfering MIMO channels. Similar to the $2 \times 2 \times$ channel, the two parallel MIMO channels are also susceptible to the effect of channel correlation and hence the AoA and AoD can be adjusted to improve system performance. A comparison with an IA based system reveals that the non IA based has a poor error performance. As a matter of fact, IA is employed in the system to improve the DoF and hence the sum rate. We further evaluate the improvement in sum rate under Rician channel.

In the second phase we consider the impact of IA based communication in Rician channel. Figure 4 shows the Sumrate as a function of SNR for a MIMO system with QPSK modulation. Considering that a configuration with 3 antennas at transmitter and receiver is considered, a single user MIMO system is formed for which the effect of Rician factor $(\kappa)$ is studied. It can be seen from Fig 4 that, in general, Sumrate increases with SNR, which is in agreement with [7]. But unlike in [7], where increasing Rician component has adverse effect on sumrate, our scheme shows an improvement in sumrate. It can also be noted that the Rayleigh component behaves like a lower bound, since there is no direct component in received signal. With $\kappa=-10 \mathrm{~dB}$, the proportion of direct component in received signal is slightly increased, and as a result, a slight improvement in capacity can be observed. Furthermore, $\kappa=0 \mathrm{~dB}$ and $10 \mathrm{~dB}$ introduces more direct component into the

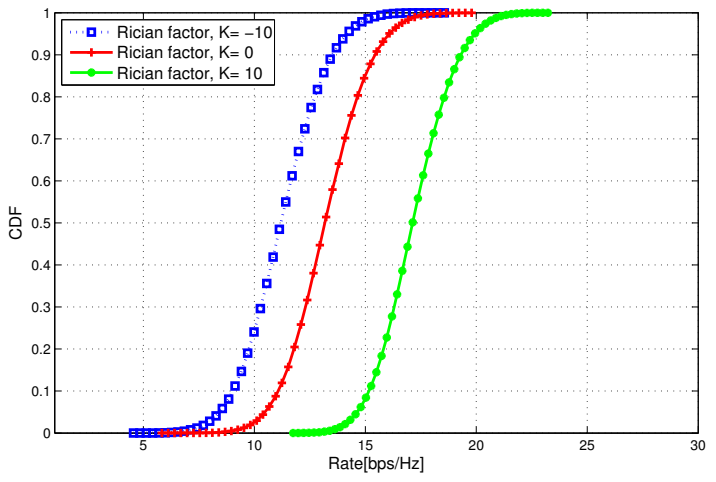

Fig. 5. CDF of channel capacity for the $2 \times 2$ MIMO, Rician channel with different Rician factor.

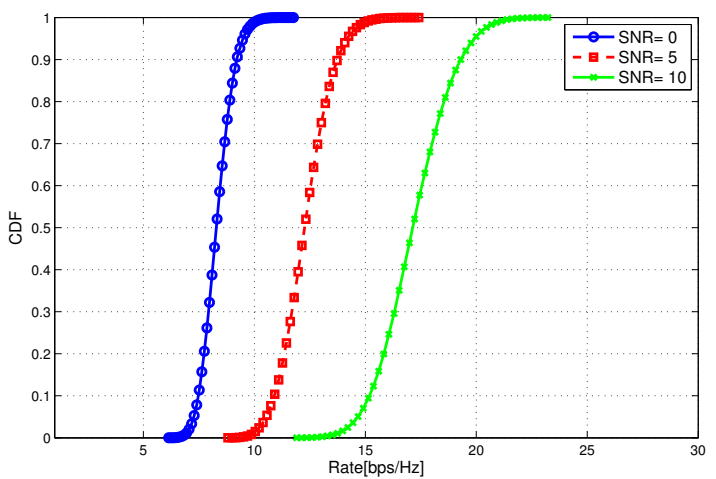

Fig. 6. CDF of channel capacity for the $2 \times 2$ MIMO, Rician channel with different SNR.

received signal, leading to significant improvement in sumrate.

The CDFs of system capacity for various SNRs and different Rician factor $(\kappa)$ under $2 \times 2 \times$ channel $(2$ HAP and 2 ground stations) configuration are shown in Fig. 6 and Fig. 5 , respectively. To analyze the effect of Rician factor on the system, we consider Rician factors $-10 \mathrm{~dB}, 0 \mathrm{~dB}$ and $10 \mathrm{~dB}$, while the SNR is fixed to $10 \mathrm{~dB}$. In 5 it is observed that the slope of the curves are similar but the median of the curves is shifted. This implies that the ergodic capacity is increased with Rician factor $\kappa$, which essentially means that the increasing LOS character improves the capacity of the system. It is also worth noting that the outage capacity is also improved with Rician factor. An SNR of $0 \mathrm{~dB}, 5 \mathrm{~dB}$ and $10 \mathrm{~dB}$ is considered to analyze the effect of SNR on the system. From Fig. 6 we can observe that the outage capacity is changed from 7.5 to 15 when SNR is varied from $0 \mathrm{~dB}$ to $10 \mathrm{~dB}$. Hence an improvement in minimum guaranteed capacity is observed with increasing SNR.

The plot of Fig 7 has the same behavior as the previous Figure, except that the system is considered as having 2 HAPs and 2 ground stations. Here, we consider two situations:

1) 2 HAPs cover common coverage area, 2 ground stations exist in this area, forming an $\mathrm{X}$ channel;

2) 2 HAPs offer service to 2 non overlapping area, forming two parallel MIMO channels. 


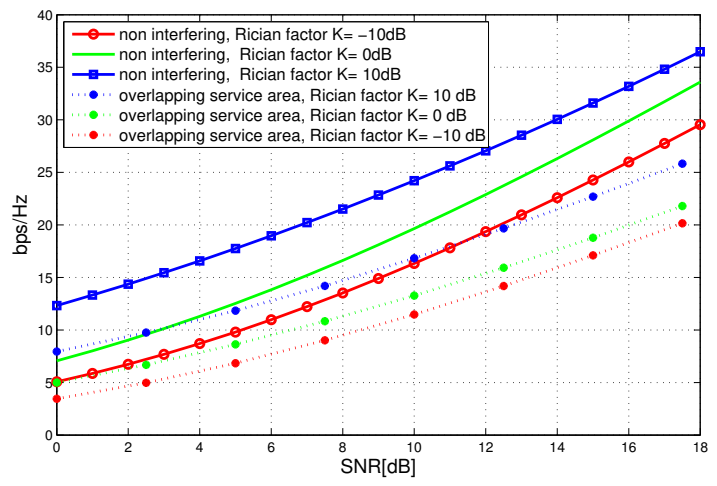

Fig. 7. Sum Rate vs SNR for $2 \times 2$ MIMO X channel for HAP based communication.

Moreover, to see what is the influence of the Rician factor $\kappa$ has on the sumrate, each line on the graph corresponds to a different value of Rician factor for the above mentioned system configurations. As a matter of fact, the second system forms an upper bound on capacity for our proposed IA based system model. Based on IA terms, the reason behind higher sumrate is that the non overlapping configuration has a DoF of 2 whereas the IA based system has only $\frac{4}{3}$. With eq (1), the reason for difference in sumrate is justified.

It can be noted that at $\kappa=-10 \mathrm{~dB}$ the difference between capacity curves for the two configurations are high. However, the difference between capacity curves at higher $\kappa$ values are reduced, the reason for such a behavior is the existence of predominant direct component in the system which behaves more like a non interfering system.

\section{CONCLUSION}

In this paper, an interference alignment scheme has been investigated for a HAP based Air-to-Ground communication system involving 2 HAPs and 2 ground stations. With the overlapped service area, the IA based communication offers significant improvement in error performance as well as in capacity. The IA based HAP communication system provides an additional advantage of having less traffic between Interplatform Links which, in turn, saves precious hardware of HAP. With the assumption of overlapped service area, our system avoids the necessity of using highly directive beamformers in HAPs. A channel with higher Rician factor $(\kappa)$ provides higher sumrate compared to lower ones. Unlike non overlapping service area based system, our system size is restricted either to two HAPs or to two ground stations. The extension of the proposed scheme to multiple HAPs is in progress.

\section{REFERENCES}

[1] Grace David, John Thornton, Guanhua Chen, George P. White, and Tim C. Tozer. "Improving the system capacity of broadband services us- ing multiple high-altitude platforms." Wireless Communications, IEEE Transactions on 4, no. 2 (2005): 700-709.

[2] F. Dong, Q. Huang, H. Li, B. Kong and W. Zhang, A novel M2M backbone network architecture, International J. of Distributed Sensor Netw., Article ID 514393, in press, 2015.

[3] A. Mohammed, A. Mehmood, F.-N.Pavlidou and M. Mohorcic, The role of high-altitude platforms (HAPs) in the global wireless connectivity, Proc. of the IEEE, vol. 99, no. 11, pp. 1939-1953, 2011.

[4] Holis Jaroslav, and Pavel Pechac. "Coexistence of terrestrial and HAP 3G networks during disaster scenarios." Radioengineering (2008).

[5] F. Dong, J. Wang, J. Yang and C. Cai, Distributed satellite cluster network: a survey, J. of Donghua University(English Edition). vol. 32, no. 2, pp.100-104, Apr. 2015.

[6] Celcer Tine, Tomaz Javornik, Mihael Mohorcic, and Gorazd Kandus. "Virtual multiple input multiple output in multiple high-altitude platform constellations." Communications, IET 3, no. 11 (2009): 17041715 .

[7] Dong Feihong, Yuanzhi He, Haitao Nan, Zongsheng Zhang, and Jingchao Wang. "System Capacity Analysis on Constellation of Interconnected HAP Networks." In Big Data and Cloud Computing (BDCloud), 2015 IEEE Fifth International Conference on, pp. 154-159. IEEE, 2015.

[8] Dong Feihong, Yuanzhi He, Haitao Nan, Zongsheng Zhang, and Jingchao Wang. "System Capacity Analysis on Constellation of Interconnected HAP Networks." In Big Data and Cloud Computing (BDCloud), 2015 IEEE Fifth International Conference on, pp. 154-159. IEEE, 2015.

[9] Grace David, and Mihael Mohorcic. Broadband Communications via High-Altitude Platforms. John Wiley \& Sons, 2011.

[10] Liu Yiming, David Grace, and Paul D. Mitchell. ”Exploiting platform diversity for GoS improvement for users with different High Altitude Platform availability." Wireless Communications, IEEE Transactions on 8, no. 1 (2009): 196-203.

[11] H. Aamir and Qamar-ul-Islam, MIMO channel modeling for integrated high altitude platforms, geostationary satellite/land mobile satellite and wireless terrestrial networks, J. of Space Technol., vol. 3, no. 1, pp. 19-26, 2013

[12] S. A. Jafar and S. Shamai, "Degrees of freedom region of the MIMO X channel," IEEE Trans. Inf. Theory, vol. 54, no. 1, pp. 151-170, Jan. 2008.

[13] Cadambe Viveck R., and Syed A. Jafar. "Interference alignment and the degrees of freedom of wireless networks." Information Theory, IEEE Transactions on 55, no. 9 (2009): 3893-3908.

[14] V. Cadambe and S. Jafar, "Interference alignment and degrees of freedom of the K-user interference channel," IEEE Trans. Inf. Theory, vol. 54, no. 8, pp. 3425-3441, Aug. 2008.

[15] S. A. Jafar, "Interference alignment: a new look at signal dimensions in a communication network," Foundations and Trends in Communication and Information Theory, vol. 7, no. 1, pp. 1-136, 2011.

[16] A. Mahmoud, M. El-Khamy, and K. Elsayed, "Interference alignment performance on MIMO X channels with imperfect channel knowledge," in Proc. Int. Workshop Signal Proc. Adv. Wireless Commun., pp. 239243, 2012.

[17] Zaji, Alenka. Mobile-to-mobile wireless channels. Artech House, 2012.

[18] Cho Yong Soo, Jaekwon Kim, Won Young Yang, and Chung G. Kang. MIMO-OFDM wireless communications with MATLAB. John Wiley and Sons, 2010. 